\title{
3D surface profile equipment for the characterization of the pavement texture - TexScan
}

\author{
João L. Vilaça ${ }^{\mathrm{a}, \mathrm{b}, *}$, Jaime C. Fonseca ${ }^{\mathrm{c}}$, A.C.M. Pinho ${ }^{\mathrm{d}}$, Elisabete Freitas ${ }^{\mathrm{e}}$ \\ ${ }^{a}$ Life and Health Sciences Research Institute, School of Health Sciences, University of Minho, 4710-057 Braga, Portugal \\ ${ }^{\mathrm{b}}$ DIGARC, Polytechnic Institute of Cávado and Ave., 4750-810 Barcelos, Portugal \\ ${ }^{\mathrm{C}}$ Industrial Electronics Department, Engineering School, University of Minho, 4800-058 Guimarães, Portugal \\ ${ }^{\mathrm{d}}$ Mechanical Engineering Department, Engineering School, University of Minho, 4800-058 Guimarães, Portugal \\ ${ }^{\mathrm{e}}$ Department of Civil Engineering, Engineering School, University of Minho, 4800-058 Guimarães, Portugal
}

\section{A R T I C L E I N F O}

\section{Article history:}

Received 25 June 2009

Accepted 15 July 2010

\section{Keywords:}

Non-contact acquisition

$3 \mathrm{D}$ reconstruction

Pavement texture characterization

Estimated Texture Depth

Texture Profile Level

\begin{abstract}
A B S T R A C T
Loads from vehicles alter the functional and structural characteristics of road pavements that directly affect the loss of resistance of the pavement and the users' comfort and safety. Those alterations require constant observation and analysis of an extensive area of road surface with high precision. For such it was developed a new scanning prototype machine capable of acquiring the 3D road surface data and characterize the road texture through two algorithms that allows calculate the Estimated Texture Depth (ETD) and Texture Profile Level (L) indicators. The experimental results obtained from nine road samples validate the developed algorithms for the texture analysis and showed good agreement between the scanning prototype equipment and the traditional Sand Patch Method.
\end{abstract}

(c) 2010 Elsevier Ltd. All rights reserved.

\section{Introduction}

Loads from vehicles alter the functional and structural characteristics of road pavements. The primary consequences are directly related not only to the loss of resistance of the pavement, but also to the users' comfort and safety. Therefore, those alterations require constant observation at a network level through compatible acquisition techniques considering high speeds on the one hand and at the project level, through techniques that allow acquiring detailed information, on the other.

Current techniques for detailed surface inspection use one spot measurement of a certain property through laser probes. The image acquisition, which is often submitted to a subsequent manual treatment, does not provide appropriate information for the establishment of 3D models of the observed surface. Achieving accurate 3D models for full-scale areas, at least as large as the tire-surface contact area, in a short period of time constitutes a step forward on the use of 3D structural analysis techniques, such as those based on Finite Element Method (FEM) and on the description of surface irregularities and defects, such as texture and segregation [1,2]. In this context, the use of inverse engineering techniques (laser trian-

\footnotetext{
* Corresponding author at: Life and Health Sciences Research Institute, School of Health Sciences, University of Minho, 4710-057 Braga, Portugal. Tel.: +351 253604881; fax: +351253510189.

E-mail address: jvilaca@ipca.pt (J.L. Vilaça).
}

gulation with laser line, among others) is more advantageous in relation to the methods used hitherto, because they allow collect a high density of data points, which combined with appropriate algorithms, allows extracting important information for road surface characterization [3-8].

The scanning prototype equipment for the acquisition of road surfaces and their texture characterization presented in this paper was developed with basis on the triangulation principle, reaching a resolution of $0.5 \mathrm{~mm}$. This needed the construction of a mechanical prototype to be used in laboratory and in field. The mechanical prototype allows the displacement of an arm which holds the measurement system over the road surface. The measurement system arm consists of two cameras and one laser line. The precision of the measurements is assured by the calibration procedure developed for the measurement system.

The proposed prototype also implied the development of a software which contains a set of algorithms that combines computer vision and computer graphics methods for: (a) 3D surface reconstruction from organized data sets (acquired data); (b) data set treatment; (c) data set analysis; (d) data set export; (e) road texture characterization, which includes two algorithms to calculate the Estimated Texture Depth (ETD) and Texture Profile Level (L) indicators.

The contribution of this investigation resides in the development of a new scanning prototype machine capable of acquiring 3D data of an extensive area of road surface with high precision, 
making possible to characterize the road texture through the use of two algorithms that allow calculating the ETD and L. The main advantages of this equipment include:

- Portability - as it can be used in laboratory and in field.

- 3D representation of a pavement.

- Storing the information in "stl (STereo Lithography)" and "txt" format - which can be read in several CAD tools or in any "txt" reader software.

- Covering a wide area, which, in the highways domain, allows for enhanced analysis using powerful software, such as the finite element method.

- The possibility to correlate the texture spectrum with other road complex parameters such as tyre-road noise, tyre-road wearing, construction quality and users comfort.

Section 2 summarizes the state-of-the-art of the existing systems which have been developed in order to characterize the texture of road surfaces.

Section 3 describes the 3D road texture acquisition system. In this section the mechanical system, the acquisition system set up and the calibration procedure used are discussed and the 3D acquisition software is presented.

Section 4 summarizes the theoretical foundations and the developed algorithms for the pavement characterization.

Finally, a comparative study between the proposed prototype equipment and a contact measurement machine (CMM) is presented in Section 5. Furthermore, the results of the texture characterization of two different types of road surfaces by using the prototype equipment are presented.

Conclusions and suggestions that aim to improve the developed system are presented in Section 6.

\section{Related work}

The functional quality of a road surface is determined by its texture and it is directly related to safety and driving comfort together with its structural quality, which is influenced by dynamic loadings. Texture also affects the environmental quality if vehicle emissions, wear and noise are considered.

The surface texture is mostly determined by the selection of the materials (especially of aggregates), the mixture design and the finishing techniques. Furthermore, surface textures change throughout time due to traffic loading and, for that reason, they must be continually monitored. Thus, within a certain texture range, monitoring techniques should allow for collecting detailed information easily at the project level, and be compatible with traffic speeds, at the network level.

Several methods to measure texture within the ranges of macrotexture and megatexture which partially fulfill those requirements have been developed.

At the network level, Si-Jie et al. [3] present a summary of the existent commercial equipments and propose a mobile imaging system that incorporates multi-modality sensors for road surface mapping and inspection applications.

At the project level, emphasis will be given to equipments developed for characterizing the texture of road surfaces, such as the Contact Measure Equipment (CMM), the Circular Texture Meter (CTM) and the RoboTex, which have the same objective as the proposed prototype equipment. This equipments and acquisition techniques go towards to a 3D description of the surface or object.

The CMM consists of a contact sensor assembled in a mechanical coordinate measure system. This data acquisition system is very precise. However, it is rather slow, expensive and restricted to materials resistant to mechanical contact [4].

The CTM and the RoboTex are examples of equipments that use computer vision techniques. Besides the fact of rapid data acquisition, computer vision allows for the acquisition of dense and precise data sets at high band widths. The CTM is a stationary apparatus that evaluates the texture profile by circular samples, presenting difficulties on texture direction interpretation [5]. The RoboTex is a robotic texture apparatus built around LMI-Selcom's line laser, acquiring texture profiling at $1 \times 1 \mathrm{~mm}$ sample intervals [6].

Image analysis techniques are based on X-ray computed tomography that convert unabsorbed X-ray to $2 \mathrm{D}$ images and then to $3 \mathrm{D}$ images. Projected Moiré Interferometry is also a highly used method [7].

These techniques represent an important advance for the acquisition of essential information useful to characterize the texture of road surfaces. Nevertheless, they are expensive and the software tools provided to characterize the pavement texture are insufficient. Thus, the development of the TexScan, a prototype equipment developed to characterize the texture of road surfaces, is intended to overcome some of the limitations presented above.

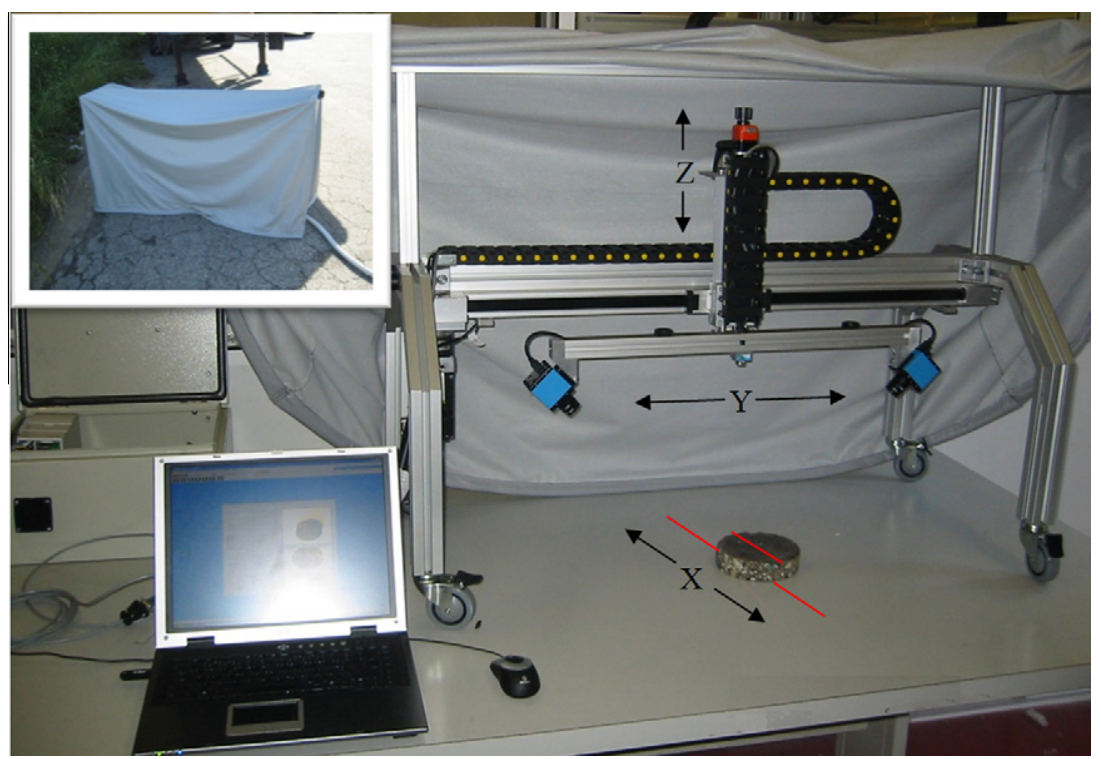

Fig. 1. 3D road surface acquisition system composed of a mechanical prototype, a data acquisition system, a motion control system and a laptop. 
Therefore, contrasting with the 3D commercial systems and in addition to the advantages presented before, the TexScan data acquisition system includes two cameras which is particularly important when dealing with open textured and porous road surfaces because occlusion zones reduce significantly. The acquisition step is variable and the lower limit is smaller than the ones of the similar equipment's $(0.5 \mathrm{~mm})$. This is an advantage because more refined analysis may be used; however the data file can reach sizes which are difficult to deal with. The data analysis software was implemented in Matlab. This feature is important because it allows
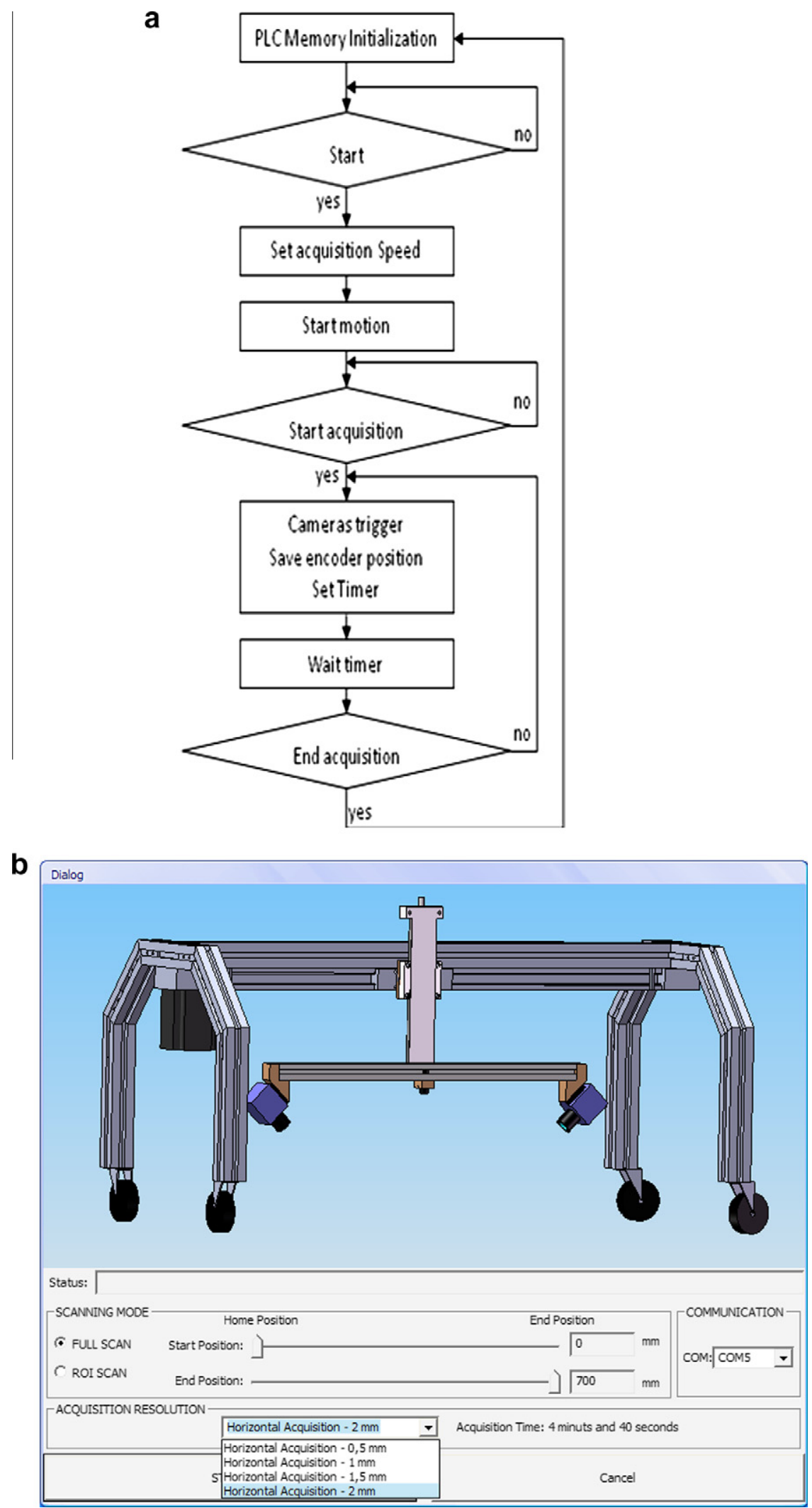

Fig. 2. (a) PLC pseudo algorithm; (b) 3D texture acquisition software: acquisition setup dialog. 
the implementation of new algorithms to perform different analysis according to user requirements.

\section{3D road texture acquisition}

The implementation of the prototype equipment comprised three main phases. In the first phase, the mechanical structure and the control system were worked out in order obtain a safe self-mobile equipment. In the second phase, a calibration method was implemented in the data acquisition system. In the last phase, a VC++ software which acquires, processes, analyzes and exports the road pavement data was developed.

\subsection{TexScan mechanical prototype and motion control system}

The mechanical prototype is constituted by two mechanical axes which move in $Z$ and $Y$ directions (Fig. 1). The function of the $Z$ axis is to set the height of the data acquisition arm (laser line and two cameras). This regulation is important to keep the road pavement surface inside the data acquisition system vision field, because laboratorial and field samples may have different heights. It is executed manually through the rotation claw coupled to the axis spindle. The function of the $Y$ axis (belt type) moves the $Z$ axis along a certain length. The maximum $Y$ course is $700 \mathrm{~mm}$, the axis is coupled to a step motor (Berger Lahr: VRDM368/50LWCOO), worked by a driver (Twin Line: TLD011F) with micro-step mode, in order to make the movement of the axis soft.

The motion of the prototype machine is controlled by a Programmable Logical Controller (PLC) (Omron: CPM1A-10CDT-VV1). The PLC controls the movements of the equipment and ensures its physical limits, disabling the equipment motion device every time that an end limit sensor is activated. Fig. 2a shows the algorithm implemented in the PLC.

The motion speed (directly associated to the system acquisition resolution) and the sample length are setups supplied by the user (Fig. 2b). The user can select the full scanning mode or the ROI scanning mode. The first one acquires data over the total acquisition length $(700 \mathrm{~mm})$. The second one executes a partial acquisition. In this mode, the user defines the starting and ending points. Relatively to the acquisition resolution, the user can select one out of four values: $0.5 \mathrm{~mm}, 1 \mathrm{~mm}, 1.5 \mathrm{~mm}$ and $2 \mathrm{~mm}$. The selection of the system resolution will directly affect the acquisition speed. These setups are sent to the PLC by the PC using the serial protocol RS232. To establish the communication the Omron Host-link protocol was implemented using $\mathrm{C}++$ language.

After setting the acquisition speed, the PLC controls the trigger signal of the two cameras. The value of the axis encoder is saved in a memory area each time that the trigger is activated.

The data acquisition system (laser and cameras) is supported by a mechanical structure built in aluminum profiles, with a base module structure assembled to free wheels, for an easier transportation. The equipment was developed to be used in laboratory and on field. It has a transportable cover which ensures adequate acquisition conditions (Fig. 1 top-left corner).

\subsection{TexScan data acquisition system}

The computer vision area includes accurate measure methods where data acquisition is made through structured light and cameras. These methods are based on geometric relations (triangulation) that can be set between the acquisition devices (cameras) and illumination (laser) and the surfaces to scan. The use of line lasers in this type of systems allows for getting high-density data (that could be programmable) and a most flexible approach for complex surfaces.
This type of methods is the basis of the data acquisition system presented hereafter. The developed system is constituted by a laser of line (lens with opening of $120^{\circ}, 3 \mathrm{~mW}$ of power and a wavelength of $735 \mathrm{~nm}$ ) and two firewire cameras (The ImagingSource: DMK 31BF03) with a resolution of $1024 \times 768$ and with $8 \mathrm{~mm}$ lenses. The use of two cameras instead of one is due to the fact that road surfaces are complex and therefore may hide the laser line visualized by the camera (occlusion problem). To minimize the effect of this problem four methods of fault in the laser line were developed: (a) constant before failure; (b) constant after the failure; (c) linear and (d) spline. These methods aim to eliminate information gaps using the information of neighboring points of the laser line to the region where the fault lies. The best results were obtained with the linear method. This method creates a line between points located immediately before and after the failure. Before proceeding to define the line validation of the points that determine it is performed. Such validation is the analysis of the points the neighborhood, checking their vertical tendency, to avoid that the line is set with noise sample points representing at the laser line.

Mechanically the acquisition arm is settled as follows: the laser is equally distant from both cameras. The cameras make a $45^{\circ}$ angle with the plane defined by the laser line. More details of the acquisition arm are shown in Fig. 3a.

Calibration this type of systems is usually a hard, time consuming task. In this specific work the calibration procedure used is the one in [9], which takes the laser plane as the calibration plan, which simplifies its implementation. The calibration procedure is divided into three steps: image horizontal calibration, image vertical calibration and image coordinates correspondence with realworld coordinates. All steps are based on polynomial interpolation
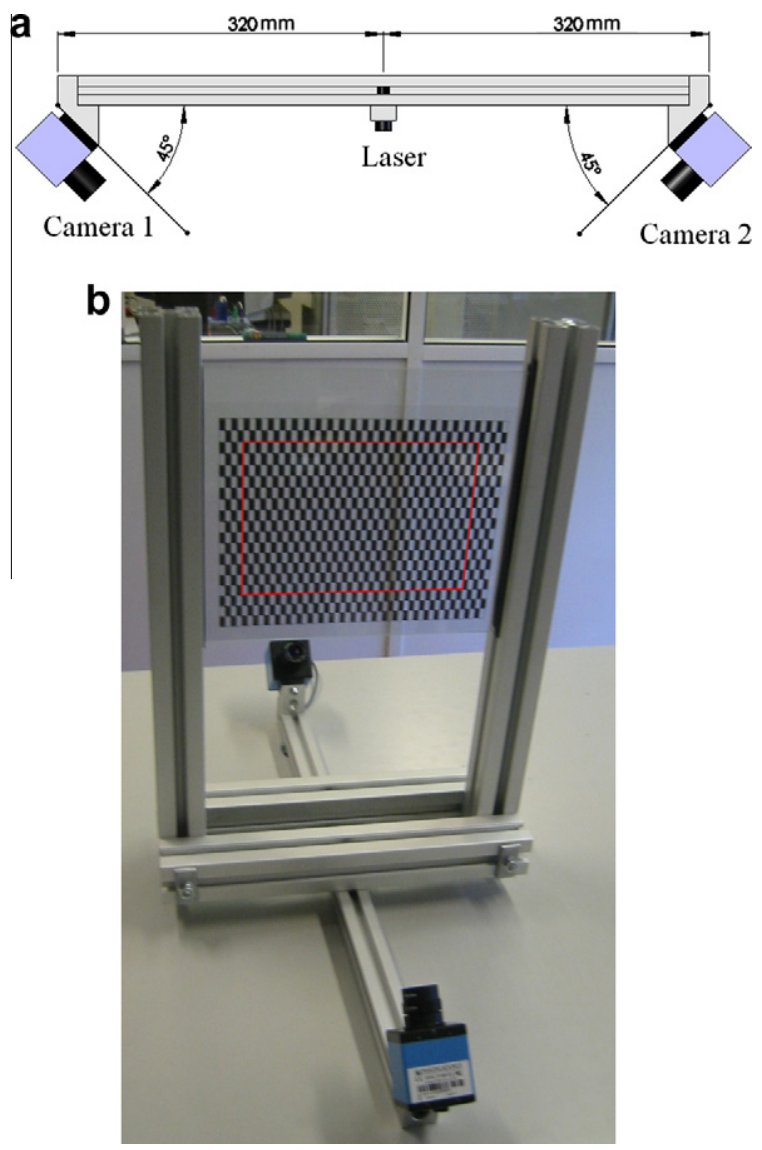

Fig. 3. Data acquisition system: (a) mechanical disposition of the acquisition arm; (b) acquisition arm assembled to the calibration support. 


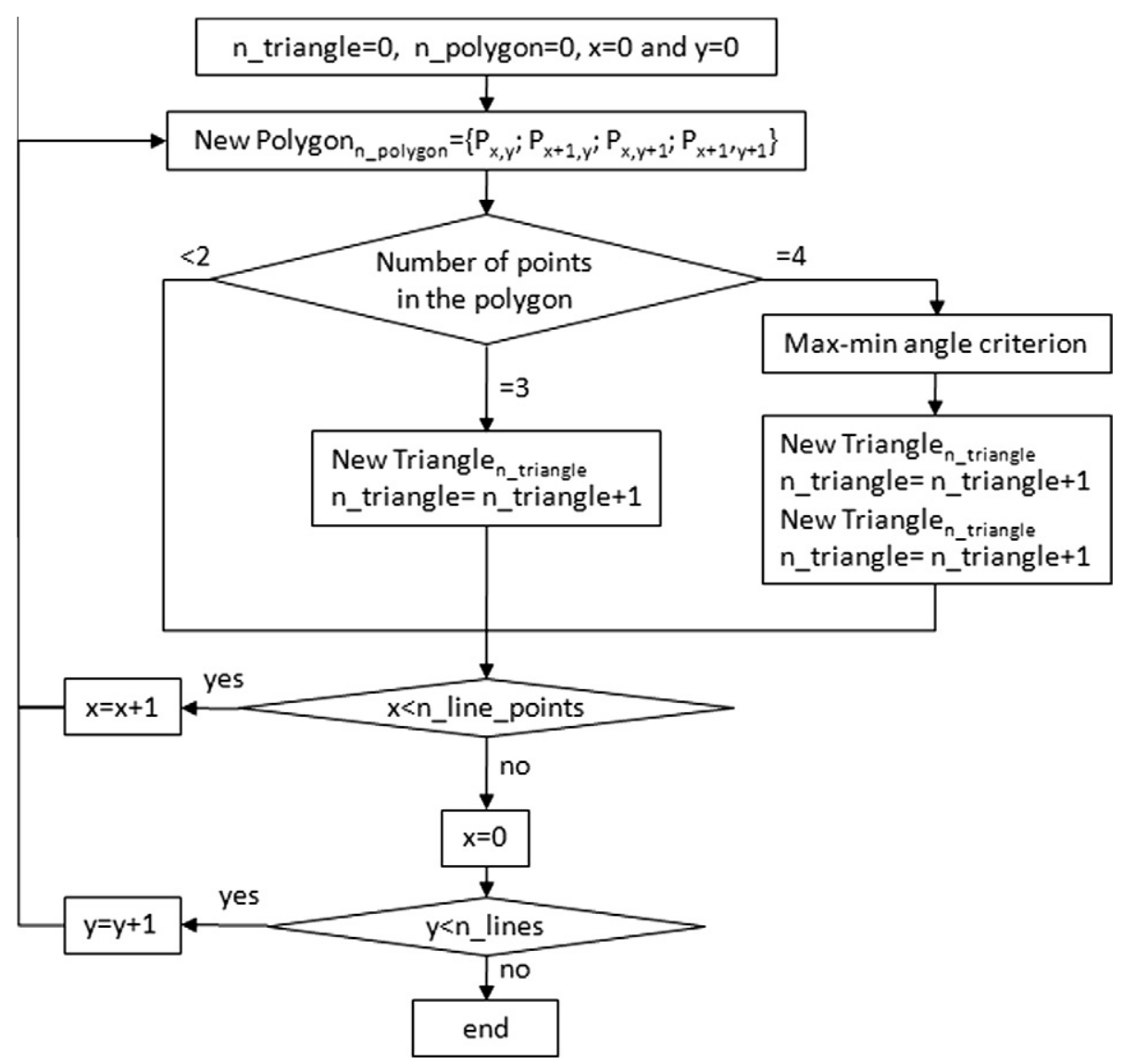

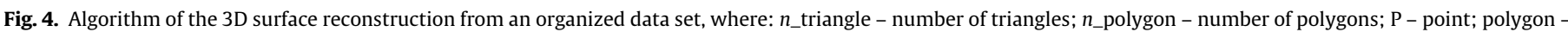

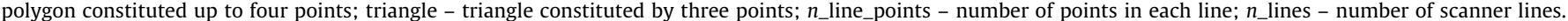

of collected pattern points (Fig. 3b). The first two steps execute the acquired images of both cameras in parallel, which results in two new images corrected horizontally and vertically. The third step is made after a logical OR between the two images that are horizontally and vertically corrected. The authors also propose an algorithm for automatic detection of laser lines based on the image vertical first derivative, which was also used.

Relatively to the system proposed in [9], the angle of both cameras was modified from $60^{\circ}$ to $45^{\circ}$ to improve the visualization of deeper areas on the surface. With this modification an improvement of the acquired data was verified. Nevertheless, the system resolution decreased, which was overcome by increasing the camera resolution. The data acquisition system vision field was defined to 200 (axis $X) \times 200$ (axis $Z$ ) $\mathrm{mm}$ with a resolution of $0.5 \times 0.5 \mathrm{~mm}$.

This software module was implemented in $\mathrm{VC}++$ and integrated in the equipment acquisition software. The software is called whenever the trigger signal of the cameras is activated by the PLC. Concisely, the algorithm calibrates and extracts the laser line view by the cameras and associates the line points to the scanner arm position (saved in the same instant of the PLC trigger shot), read from a PLC memory position.

\subsection{TextScan software}

The software of the TexScan was implemented in VC++. It is responsible for the following tasks: data acquisition, data processing, data analysis and data export for the formats .txt and .stl (STereo Lithography).

Data are acquired line by line. Each line is constituted by a set of points and represents 2D height map ( $z$ and $x$ coordinates) of the acquired sample. The result of the acquisition process is a $3 \mathrm{D}$ height map, where the $y$ coordinate of each line is given by the axis encoder value.

Once the acquired data are organized in space, the developed software uses an algorithm (Fig. 4) to produce a triangular mesh. This algorithm uses the max-min angle criterion [10] for setting triangles from a quadrangular polygon.

The software provides three types of mesh smoothing filters for data processing. Mesh smoothing is a technique that adjusts the point-coordinates of a data set. The objective of the mesh smoothing is the improvement of the appearance of a mesh and/or the improvement of the shape of the data set. The most used smoothing technique is the basic Laplacian smoothing one, which is based on the Laplacian flow. The discrete implementation is achieved by multiplying the Laplacian operator mask with a $3 \times 3$ mask (Fig. 5a) to the data set. Based on this technique a set of smoothing

\begin{tabular}{|l|l|l|}
\hline $\mathbf{a}$ & 0.2 & 0 \\
\hline 0.2 & -1.8 & 0.2 \\
\hline 0 & 0.2 & 0 \\
\hline
\end{tabular}

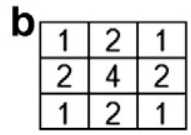

\begin{tabular}{|l|l|l|l|l|}
\hline 2 & 7 & 12 & 7 & 2 \\
\hline 7 & 31 & 52 & 31 & 7 \\
\hline 12 & 52 & 127 & 52 & 12 \\
\hline 7 & 31 & 52 & 31 & 7 \\
\hline 2 & 7 & 12 & 7 & 2 \\
\hline
\end{tabular}

d

\begin{tabular}{|l|l|l|}
\hline 1 & 1 & 1 \\
\hline 1 & 1 & 1 \\
\hline 1 & 1 & 1 \\
\hline
\end{tabular}

Fig. 5. Smoothing masks: (a) laplacian $3 \times 3$, (b) Gaussian $3 \times 3$, (c) Gaussian $5 \times 5$ and $(\mathrm{d})$ average $3 \times 3$. 


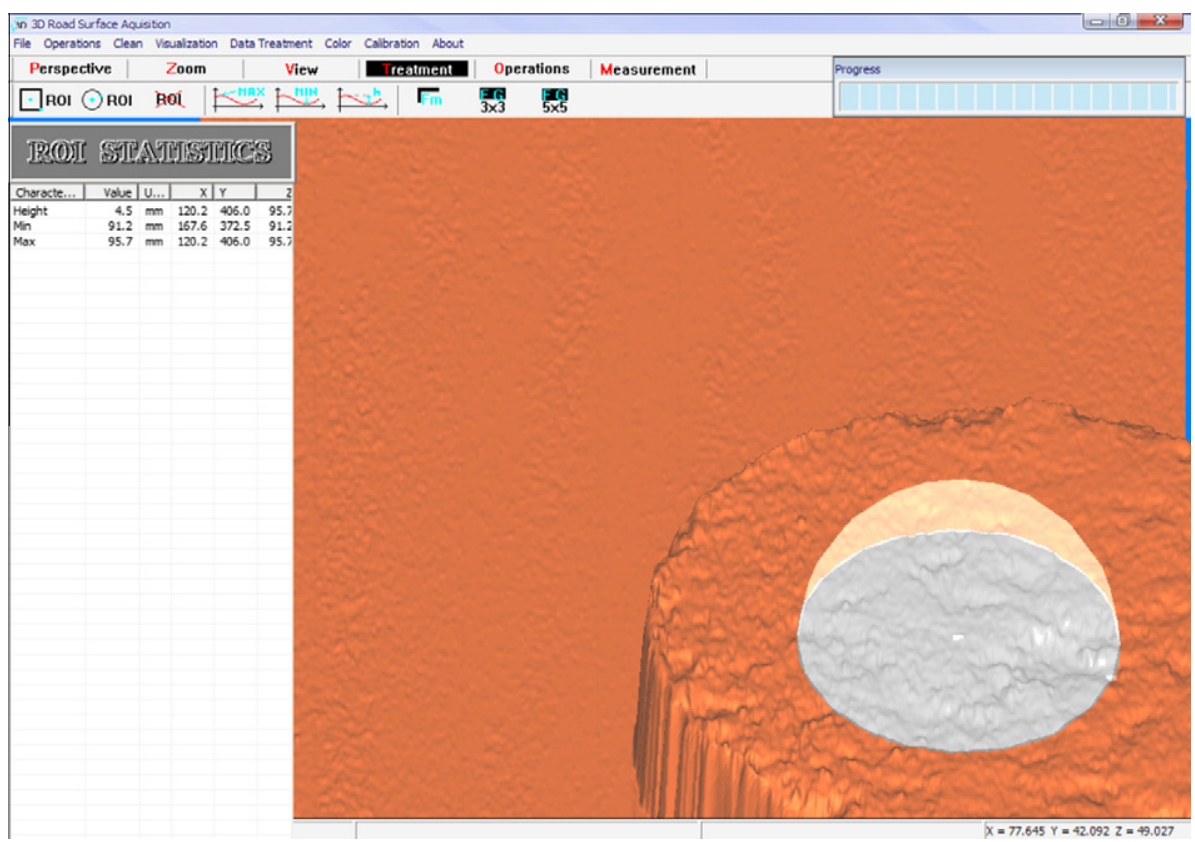

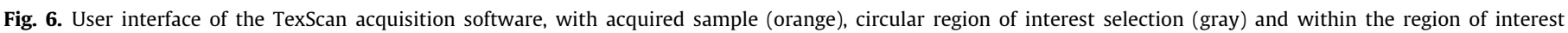
maximum, minimum and height values (bottom-left corner of the image).

surface filters were developed. A Gaussian filter with $3 \times 3$ (Fig. 5b) and $5 \times 5$ (Fig. $5 \mathrm{c}$ ) neighboring and a simple $3 \times 3$ average filter (Fig. 5d) were implemented.

To preserve the data set topology, a normal vector analysis is executed in each neighborhood of the analyzed point. If the point in analysis presents neighborhood normal vectors with different orientations (this difference should be higher than $30^{\circ}$ ) the smoothing technique is not applied to the point. The filter which showed the best results was the Gaussian $5 \times 5$ one.

Furthermore, this software provides the user with the possibility of selecting a region of interest (circular or rectangular), of the acquired sample for data analysis (maximal, minimal and height values) and data export (Fig. 6). In relation to the data export, the software disposes of two export formats: STL neutral file and TXT file. The STL file can be used to model the road by usinf a finite element analysis software. The TXT file is used to characterize the road texture in the software developed in Matlab, which is presented in the next section. The .txt file is saved with all the point lines of the acquired sample belonging to the select region of interest.

\section{3D road texture characterization}

The road surface texture has been measured worldwide for many years by the so-called "sand-patch method". It relies on a given volume of sand which is spread out on a road surface. Recently the sand was replaced by glass spheres. The sand, or the glass spheres, is distributed to form a circular patch, the diameter of which is measured [11]. By dividing the volume of sand with the area covered, a value is obtained which represents the average depth of the sand layer, i.e. an average "texture depth". The results of this method depend on many factors such as the operator or the volume of the material used. An uncertainty of $0.227 \mathrm{~mm}$ is associated to this method.

At the present time, many procedures have been developed to determine the Mean Profile Depth, which are based on the acquisition of the profile of the surface, as previously described.

The profile of a road surface may be described by two coordinates: the distance along a certain travel direction, the amplitude which is normal to the surface plane [12] and the texture wavelength defined as the (minimum) distance between periodically repeated parts of the curve.

After the acquisition of the set of profiles, which constitutes a 3D sample of the pavement, it is necessary to characterize them with appropriate indicators, such as the Mean Profile Depth (MPD) and the Texture Profile Level (L), using different and complementary mathematical techniques. Some of these mathematical techniques are based on the Fourier technique and filtering processes (Texture Profile Level) in order to determine the amplitude of its spectral components (wavelengths or spatial frequencies). They were implemented in Matlab version 7.1 using .m files [13]. The whole Matlab processing is made by lines which are composed by the points obtained after the acquisition and processing effectuated by the acquisition software of the prototype machine.

In this section the theoretical foundations and the developed algorithms for the pavement characterization were summarized.

\subsection{Mean Profile Depth and Estimated Texture Depth}

The depth of a profile is the difference in height between the profile and a horizontal line through the top of the highest peak (the peak level) within a distance along the surface of the same order of length as a typical tire-pavement interface. The Mean Profile Depth (MPD) is calculated at a certain profile distance (base line), usually $10 \mathrm{~cm}$, as indicated in Fig. 7 and by Eq. (1) [11].

$\mathrm{MPD}=\frac{\text { Peak level }(1 \mathrm{st})+\text { Peak level }(2 \mathrm{nd})}{2}-$ Average level

For comparison reasons with volumetric techniques, such as the "Sand Patch Test", the MPD may be transformed through a conversion into an ETD (Estimated Texture Depth) as shown in Eq. (2) [11].

$\mathrm{ETD}=0.2+0.8 \times \mathrm{MPD}$

The ETD conversion expression was determined to be applied on data coming from laser profilometers. Further details can be found in [15]. 


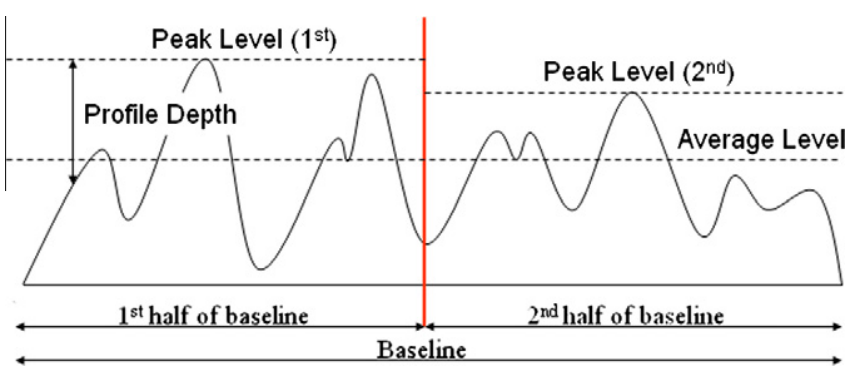

Fig. 7. Illustration of the base line concept, peak level, profile depth and average level.

The validity of the conversion expression for the equipment under analysis is addressed here because texture specifications used by road administrations are still based on volumetric techniques, despite the increasing use of laser based techniques.

With this system the base lines are set in each scanned line, the number of which depends on the length of the sample. Therefore, the MPD of the sample is determined by averaging the Mean Profile Depth calculated for each line through Eq. (1). The ETD is calculated through Eq. (2) (see Fig. 8).

\subsection{Texture Profile Level}

Whenever a surface profile is defined by its spectrum, the resulting amplitudes within a certain band may be transformed into a unique indicator defined as Texture Profile Level $(\mathrm{L})$. The Texture Profile Level is a logarithmic transformation of an amplitude representation of a profile, as shown in Eq. (3) [16].

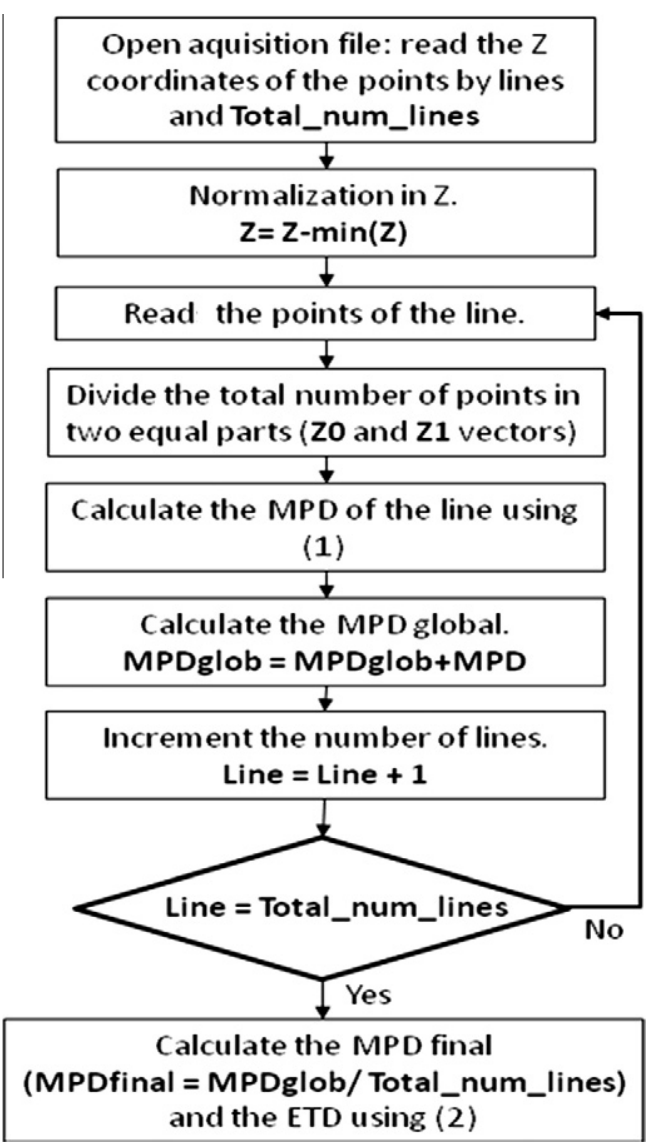

Fig. 8. Algorithm for ETD calculation.

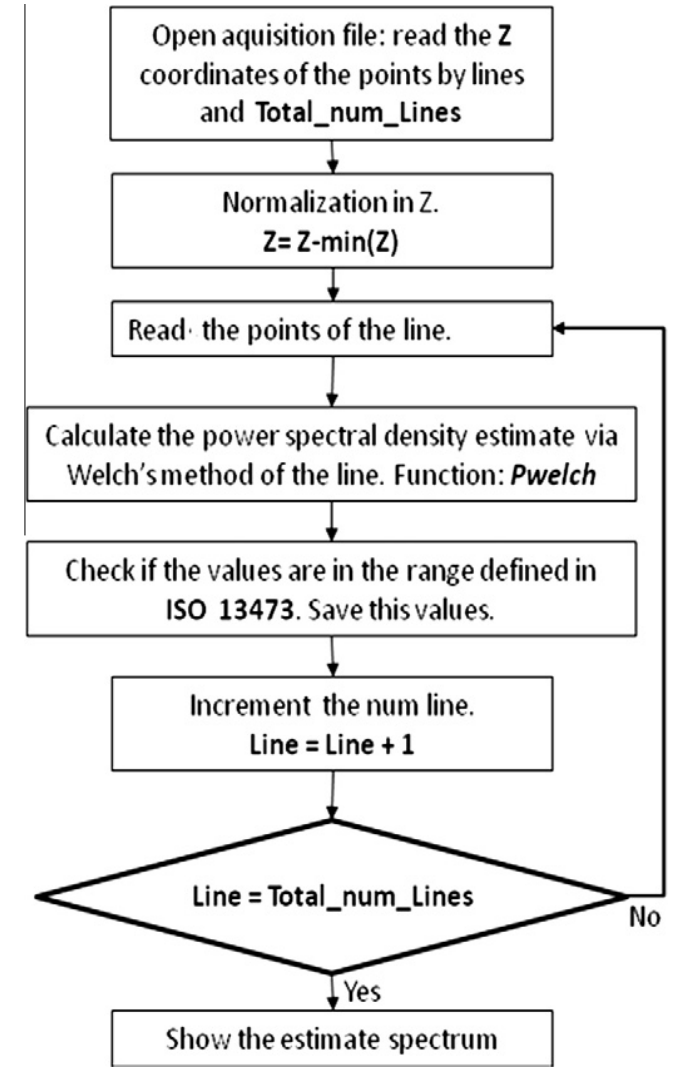

Fig. 9. Algorithm for Texture Profile Level visualization.

$L_{t x, \lambda} \quad$ or $\quad L_{T X, \lambda}=20 \log \frac{a_{\lambda}}{a_{\text {ref }}}$

where $L_{t x, \lambda}$ is the Texture Profile Level in one-third-octave bands (re $10^{-6} \mathrm{~m}$, in $\mathrm{dB}$ ); $L_{T X, \lambda}$ is the Texture Profile Level in octave bands (re $10^{-6} \mathrm{~m}$ in $\mathrm{dB}$ ); $a_{\lambda}$ is the root mean square value of the surface profile amplitude (in $\mathrm{m}$ ); $a_{\text {ref }}$ is the reference value $=10^{-6} \mathrm{~m} ; \lambda$ is the subscript that indicates the value obtained with a one-third-octaveband filter or octave-bad-filter having centre wavelength $\lambda$.

If the power spectral density (PSD) from the results of a Discrete Fourier Transform (DFT) of the profile is calculated, the fractionaloctave-band texture level is calculated with:

$L_{t x, m}=10 \log \frac{Z_{p, m}}{a_{\mathrm{ref}}^{2}}$

where $a_{\text {ref }}$ is the reference root mean square value of the surface profile amplitude $=10^{-6} \mathrm{~m} ; L_{t x, m}$ is the (texture) profile level within fractional-octave-band $m$ in $\mathrm{dB}$ re $10^{-6} \mathrm{~m} ; Z_{p, m}$ is the power within the fractional-octave-band $m$, calculated from the narrow band power spectral density.

According to Standard ISO 13,473-1 [14], the ranges of texture that may be covered by this equipment are within macrotexture and megatexture ranges. They are defined as the deviation of a pavement surface from a true planar surface with the characteristic dimensions along the surface of $0.5-50 \mathrm{~mm}$ and peak-to-peak amplitudes in the range of $0.1-20 \mathrm{~mm}$ for macrotexture and 50 $500 \mathrm{~mm}$ and peak-to-peak amplitudes in the range of $0.1-50 \mathrm{~mm}$, respectively, for megatexture. The implemented algorithm is depicted in Fig. 9.

\section{Experimental results}

To demonstrate the capabilities of the proposed prototype machine, two types of analysis were effectuated. The first one is 


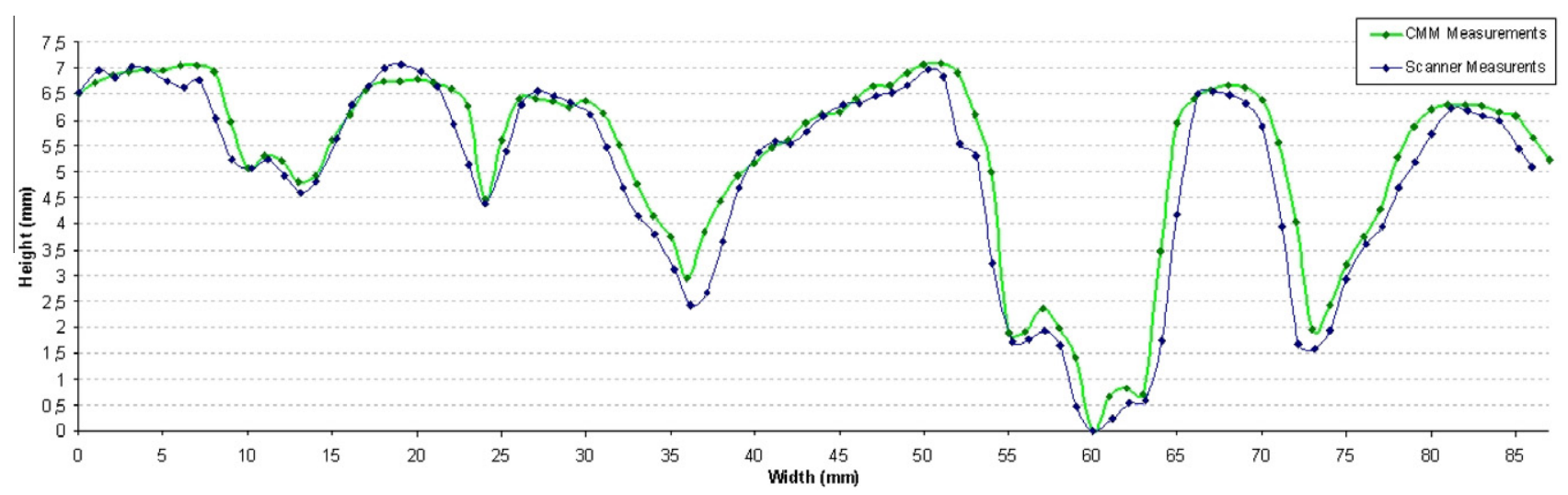

Fig. 10. This graph establishes a comparison between the lines whose points were acquired by the acquisition system used and the CMM over road profile surface.

intended to certify the acquisition system of the prototype machine equipment. For this analysis, a sample of road pavement was used for comparing the results obtained by the prototype machine acquisition system and by a high precision CMM (Mitutoyo, Class 0 , Precision $0.1 \mu \mathrm{m}$ ). The second analysis presents the characterization of two types of road surface textures, through the determination of the Mean Profile Depth (MPD) and of the Texture Profile Level (L).

\subsection{Data acquisition system error results}

The possibility of a practical application of the developed acquisition system in the dimensional inspection of road surfaces, regarding macrotexture and megatexture, is demonstrated by means of a comparative study of a profile line carried out with a CMM machine and the acquisition system (Fig. 10) [9]. The horizontal sampling was carried out at every $1 \mathrm{~mm}$. The procedure consisted of drawing a line in the sample to define the same path for both the CMM and the developed acquisition system.

The sample collected has a complex profile which constitutes a drawback to the profile acquisition in some narrow concavities captured by the CMM machine, as shown at intervals: $\{23,28\}$ and $\{30,40\}$. Tables 1 and 2 show the statistical comparison between the two acquisition systems. It is possible to draw the following conclusions:

- The mean value of the CMM system $(0.36 \mathrm{~mm})$ is higher than the mean value of the developed system. This fact may be the consequence of two factors: (a) an error made by the acquisition system; (b) CMM difficulty in reaching narrow concavities.

- The maximal vertical value of acquisition points has almost the same value for both systems. The same happens for the minimal vertical value.

Table 1

Comparison between points acquired by CMM and the acquisition system used

\begin{tabular}{lllll}
\hline Measurement system & Mean & Min & Max & Std. \\
\hline CMM (unit: mm) & 5.29 & 0.00 & 7.09 & 1.76 \\
Developed system (unit: $\mathrm{mm}$ ) & 4.93 & 0.01 & 7.08 & 1.92 \\
\hline
\end{tabular}

Table 2

Errors in distances between points acquired by CMM and the developed system.

\begin{tabular}{lllll}
\hline Measurement & Mean & Min & Max & Std. \\
\hline Error (unit: $\mathrm{mm}$ ) & -0.13 & -0.24 & 0.05 & 0.07 \\
\hline
\end{tabular}

- The maximal acquisition point has almost the same value for both systems.

- The standard deviation is bigger in the developed prototype equipment than in the CMM system; this fact could be influenced by the same factors that influenced the mean value.

\subsection{Road texture characterization of pavement surfaces}

The objective of the second analysis is the characterization of the macrotexture of nine road surface samples using the developed prototype machine and the comparison of the obtained ETD values with the values calculated by the "Sand Patch Method". According to road surface specifications, the profile depth of the samples chosen characterize the surface layers used in the country. These samples can be grouped into three layer types which can be divided according to the texture type in smooth and rough as follows:

- Porous asphalt, smooth (samples A and B).

- Dense asphalt, smooth (samples C and D).

- Dense asphalt, rough (sample E).

- Open graded asphalt, smooth (samples F and G).

- Open graded asphalt, rough (sample H).

- Porous asphalt, rough (sample I).

The nine samples were acquired by the developed prototype equipment using a precision of $0.5 \times 0.5 \times 0.5 \mathrm{~mm}$. A lower precision could have been chosen, for example that of $1.0 \times 0.5 \times 0.5 \mathrm{~mm}$, but if the diameter of the glass beads with a maximum of $0.25 \mathrm{~mm}$ is considered relevant data could be discarded. After acquiring the samples, a circular region of interest (ROI) of $120 \mathrm{~mm}$ (diameter) was created in each sample. The diameter and centre of the selected ROI was approximately the same that was used in the "Sand Patch Method". The points that belonged to the selected ROI were then exported to be used in Matlab.

Fig. 11 presents the aspect and the 3D acquired surface (represented by a color height map) of four surfaces which were chosen to illustrate possible differences that might be found on this type of materials.

The MTD and ETD values of the nine samples calculated by the developed prototype equipment and by the "Sand Patch Method" are presented in Table 3. In addition to that, the number of points of which samples are composed and the MPD and ETD calculated by transposing the data matrix are presented. The comparison of the results obtained in the scanning direction and orthogonally to that direction permit validating the adaption of the method recommended in [11] to 3D surfaces. 
a

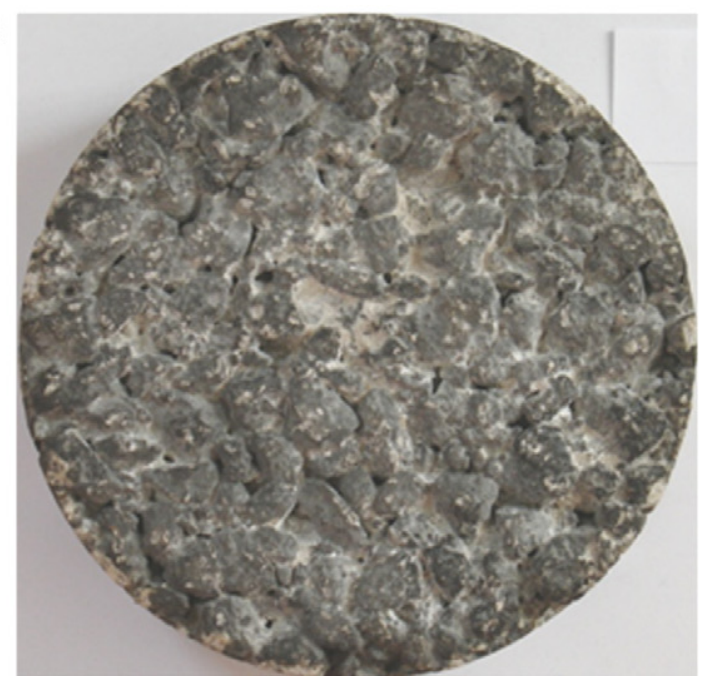

C

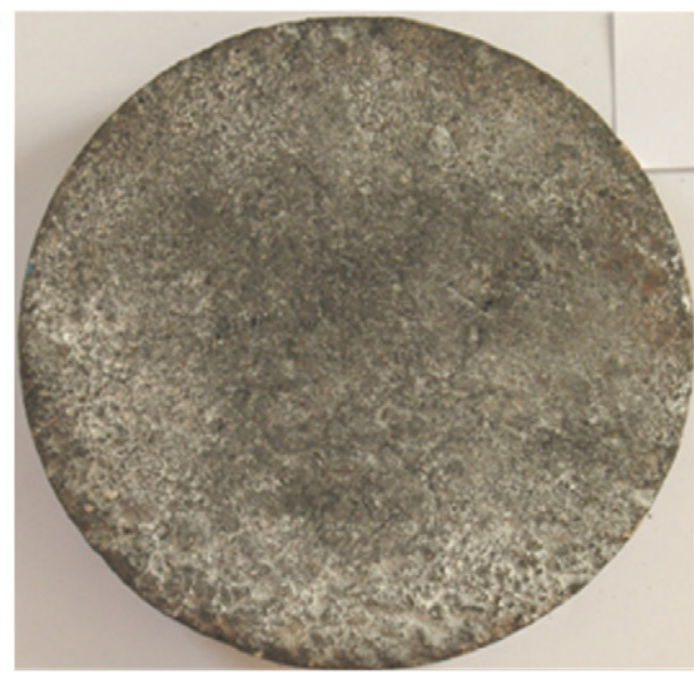

e

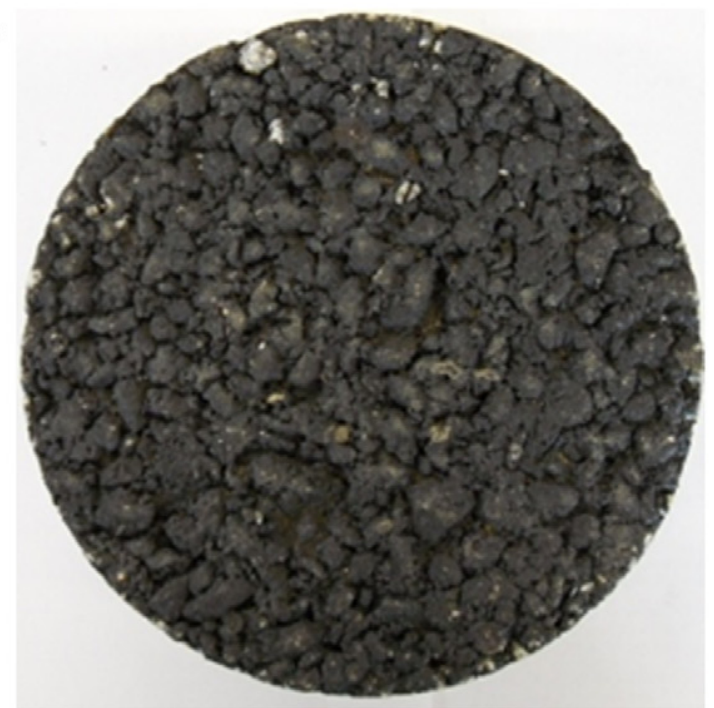

b

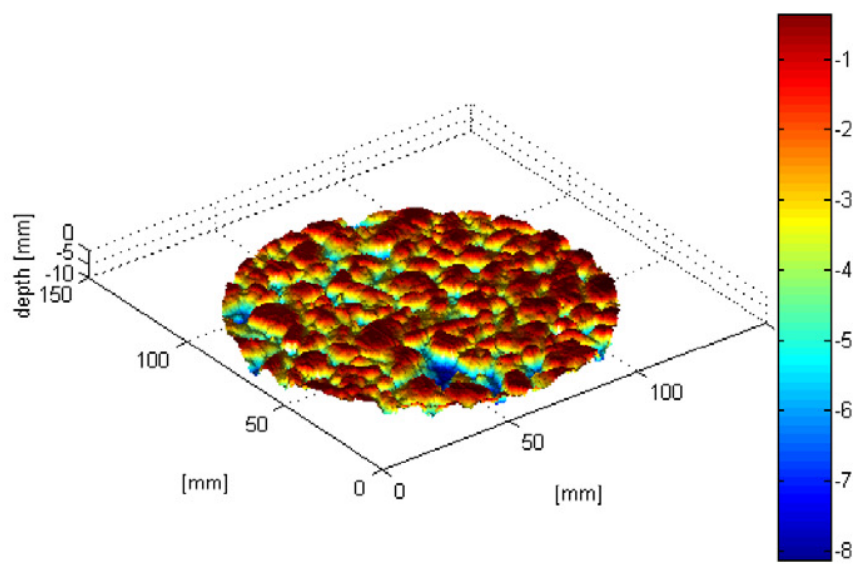

d

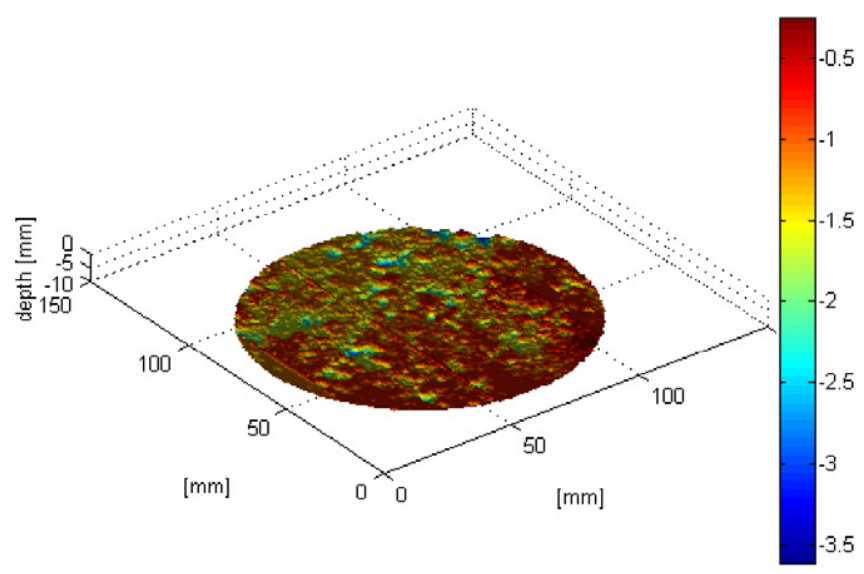

f

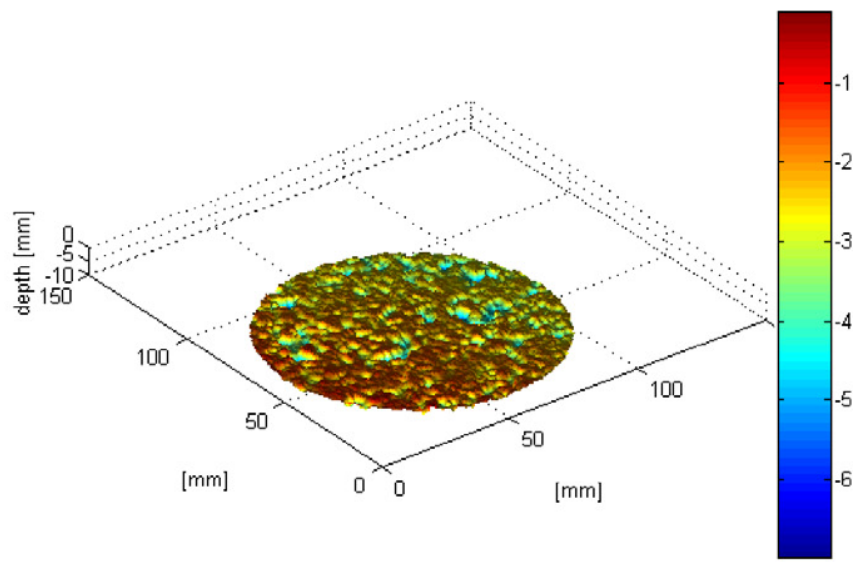

Fig. 11. Samples photography and corresponding 3D acquired surface: (a and b) - sample A; (c and d) - sample D; (e and f) - sample G; (g and h) - sample I.

The results show that the ETD values calculated by the TexScan are similar to the ones calculated by the "Sand Patch Method" in both directions, as shown in Table 4 . Likewise, the relation be- tween the ETD values calculated by the developed prototype equipment and the ETD values calculated by the "Sand Patch Method" are practically the same, varying more in samples $C$ and 


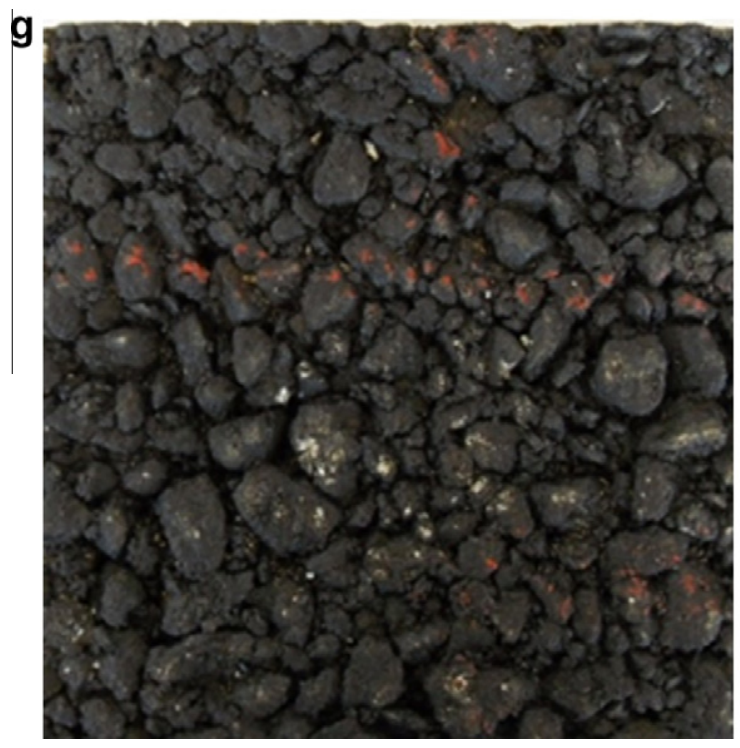

h

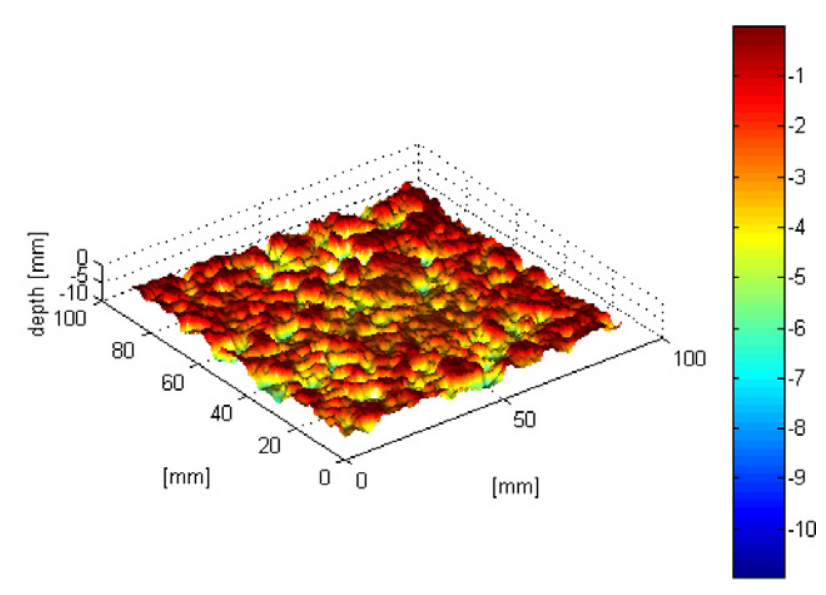

Fig. 11 (continued)

D in the orthogonal direction. This fact may be explained by the difficulty in applying the "Sand Patch Method" in this type of samples because they have extreme values of MPD. The results obtained validate the use of the conversion expression presented in Eq. (2) and the developed algorithm for this indicator in texture characterization.

Fig. 12 depicts the Texture Profile Levels versus the spatial frequency of the four samples chosen as example. By analyzing the Texture Profile Level charts, it is possible to verify that the frequency values of each sample are approximately the same. Nevertheless, for each frequency, the texture level differentiates the samples, which is an advantage if the method is compared to others that do not provide this parameter. Furthermore, this method can be applied on considerably large areas, while other available techniques that provide the same parameter are used with very small areas (e.g. $2 \mathrm{~cm}^{2}$ ) or with only one scanned line. This shows that it will be possible to use the developed algorithm to characterize the texture of a large area by visualizing the surface of the road easily and by analyzing which frequencies suffer significant changes and use this information afterwards for other enhanced analysis.

\section{Conclusions and future work}

This paper presented different construction stages of a prototype equipment to obtain the irregularities of a road pavement surface and its texture characterization in $3 \mathrm{D}$.

A new mechanical configuration of the acquisition arm was adapted and arm components with different characteristics, com-

Table 3

MPD and ETD results.

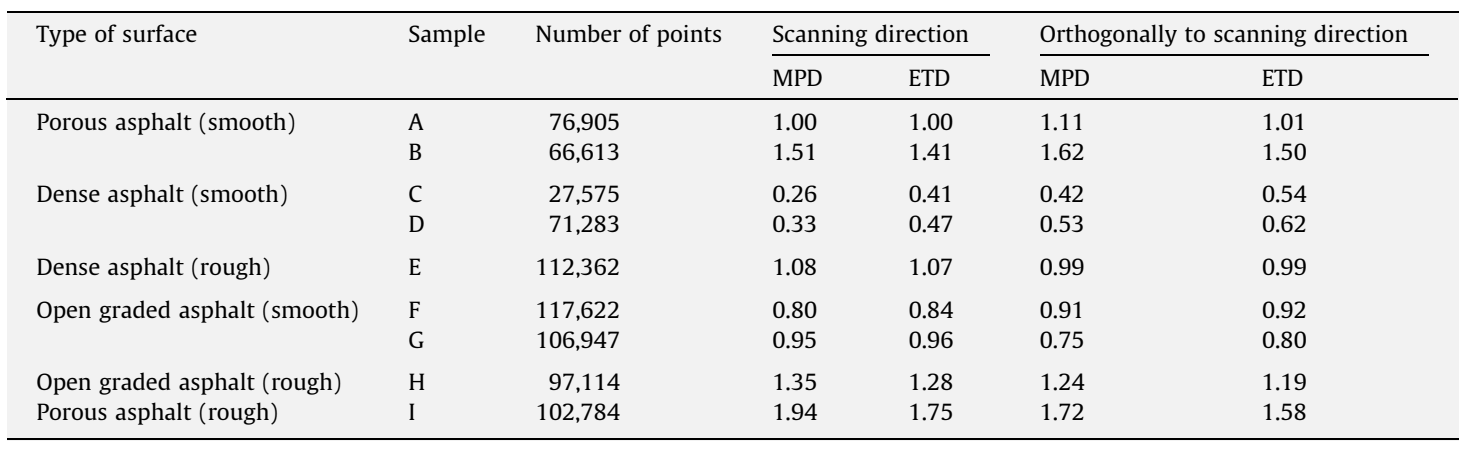

Table 4

Relation between ETD of the prototype equipment and Sand Patch Method.

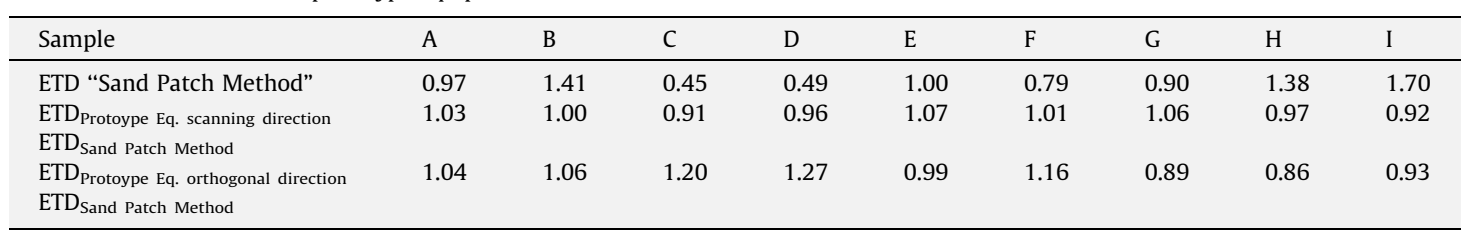



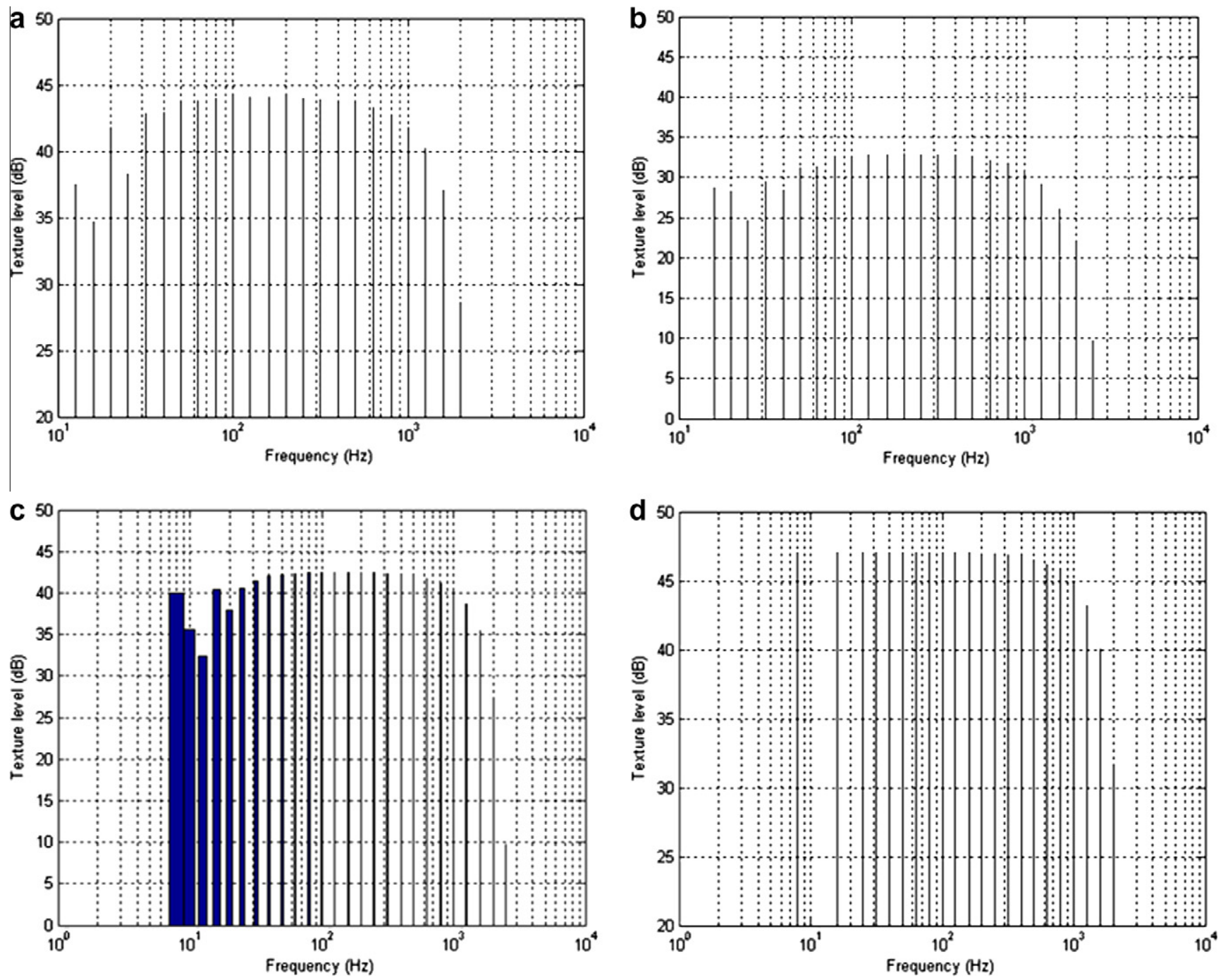

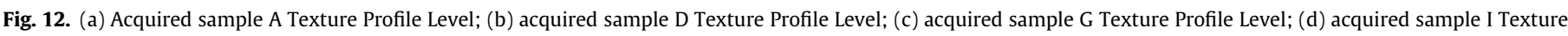
Profile Level.

paratively to the system presented by Vilaça et al., were used in a way to improve the acquisition of difficult visualization areas, that this type of surfaces present.

In the pavement characterization module, two algorithms were developed: one for the calculation of the ETD and another one for the determination of the Texture Profile Level. Both algorithms were tested over two different types of road surfaces. The results validated the use of these algorithms to characterize the texture using the ETD and Texture Profile Level indicators.

The machine also comprises a simple and friendly user interface, as a result of the interconnection among the machine modules and the low acquisition times. A road sample of $700 \mathrm{~mm}$ length for $200 \mathrm{~mm}$ width, with a resolution of $0.5 \times 0.5 \mathrm{~mm}$, was acquired and post-processed in $280 \mathrm{~s}$.

The experimental results obtained from nine road samples validate the developed algorithms for the texture analysis and showed good agreement between the scanning prototype equipment and the traditional Sand Patch Method.

Further developments from this work will include providing the machine with a module for the characterization of cracks that may develop on the road surface. Thus, the equipment will be able to determine the crack length, crack orientation, crack depth and maximum and medium crack opening.

\section{Acknowledgments}

This work has been supported by the "Fundação para a Ciência e a Tecnologia" (Portugal) through the PhD Grant referenced SFRH/ $\mathrm{BD} / 18155 / 2004$.

\section{References}

[1] Merzouki R, Ould-Bouamama B, Djeziri MA, Bouteldja M. Modelling estimation of tire-road longitudinal impact efforts using bond graph approach Mechatronics 2007;17(2-3):93-108.

[2] Gruber Steffen, Semsch Martin, Strothjohann Thomas, Breuer Bert. Elements of a mechatronic vehicle corner. Mechatronics 2002;12(8):1069-80.

[3] Yu Si-Jie, Sukumar Sreenivas R, Koschan Andreas F, Page David L, Abidi Mongi A. 3D reconstruction of road surfaces using an integrated multi-sensory approach. Opt Lasers Eng 2007;45(7):808-18.

[4] Woźniak A, Dobosz M. Metrological feasibilities of CMM touch trigger probes. Part I: 3D theoretical model of probe pretravel. Measurement 2003;34(4):273-86.

[5] Hanson Douglas I, Prowell Brian D. Evaluation of circular texture meter for measuring surface texture of pavements. NCAT report 04-05; September 2004.

[6] TCPSC newlatter. The Transtec center for pavement surface characteristics, vol. 1(2). A Division of The Transtec Group Inc.; 2006.

[7] Abbas Ala, Emin Kutay M, Azari Haleh, Rasmussen Robert. Three-dimensional surface texture characterization of portland cement concrete pavements. Comput-Aid Civil Infrastruct Eng 2007;22(3):197-209. 
[8] Indyk Daniel, Velastin Sergio A. Survey of range vision systems. Mechatronics 1994;4(4):417-49.

[9] Vilaca Joao L, Fonseca Jaime C, Pinho Antonio M. Calibration procedure for 3D measurement systems using two cameras and a laser line. Opt Laser Techno 2009;41(2):112-9.

[10] Choi BK, Shin HY, Yoon YI, Lee JW. Triangulation of scattered data in 3D space. Comput-Aid Des 1988;20(5):239-48.

11] ASTM E965-96. Standard test method for measuring pavement macrotexture depth using a volumetric technique. West Conshohocken: American Society for Testing and Materials; 2006

[12] ISO/CD 13473-5. Characterization of pavement texture by use of surface profiles - part 5: measurement of megatexture. Draft standard ISO/CD 134735. Geneve, Switzerland: International Organisation for Standardisation (ISO); 2009
[13] MATLAB 7. Function reference: Volume 1 (A-E), Volume 2 (F-O), Volume 3 (PZ). Natick (MA): The MathWorks, Inc.; September 2007.

[14] ISO 13473-1. Characterization of pavement texture by use of surface profiles part 1: determination of mean profile depth. Geneve, Switzerland: International Organisation for Standardisation (ISO); 1997.

[15] Wambold JC, Antle CE, Henry JJ, Rado Z, Descornet G, Sandberg U, et al. International PIARC experiment to compare and harmonize texture and skid resistance measurement. Final report, no. 01.04.T, to the technical committee on surface characteristics. Paris: World Road Association (PIARC); 1995.

[16] ISO/CD 13473-4. Characterization of pavement texture by use of surface profiles - part 4: spectral analysis of surface profiles. Draft standard ISO/CD 13473-4. Geneve, Switzerland: International Organisation for Standardisation (ISO); 2008. 